\title{
Classical and Quantized Affine Physics: A Step towards it
}

\author{
Jan J. SEAWIANOWSKI ${ }^{\dagger}$ and Vasyl KOVALCHUK ${ }^{\ddagger}$ \\ Institute of Fundamental Technological Research, Polish Academy of Sciences, \\ Świętokrzyska 21, 00-049 Warsaw, Poland \\ E-mails: ${ }^{\dagger}$ jslawian@ippt.gov.pl, ${ }^{\ddagger} v k o v a l @ i p p t . g o v . p l$
}

This article is part of the Proceedings titled "Geometrical Mathods in Physics: Bialowieza XXI and XXII"

\begin{abstract}
The classical and quantum mechanics of systems on Lie groups and their homogeneous spaces are described. The special stress is laid on the dynamics of deformable bodies and the mutual coupling between rotations and deformations. Deformative modes are discretized, i.e., it is assumed that the relevant degrees of freedom are controlled by a finite number of parameters. We concentrate on the situation when the effective configuration space is identical with affine group (affinely-rigid bodies). The special attention is paid to left- and right-invariant geodetic systems, when there is no potential term and the metric tensor underlying the kinetic energy form is invariant under left or/and right regular translations on the group. The dynamics of elastic vibrations may be encoded in this way in the very form of kinetic energy. Although special attention is paid to invariant geodetic systems, the potential case is also taken into account.
\end{abstract}

\section{Introduction}

Typical physical theories are based on groups preserving bilinear or sesquilinear forms. These forms are fixed and belong to the absolute, non-dynamical sector of the theory. Typical examples are Euclidean and pseudo-Euclidean scalar products in space or spacetime. In field theory and quantum physics one deals with unitary and pseudo-unitary groups preserving Hermitian scalar products in target spaces of field multiplets. In tetrad models of gravitation the internal space is again endowed with Minkowskian geometry. It is something else different from "external" geometry of special relativity; internal Lorentz group which rules it must not by mixed up with the external group of special relativity.

External and internal metrics are machines contracting tensor indices and enabling us to build scalars and scalar densities necessary for constructing Lagrangians. The resulting models are linear or weakly (perturbatively) nonlinear, in particular, scalars and densities quadratic in dynamical variables do exist. But it is clear that the affine (Tales) geometry is mathematically more primary. Does a hypothetical affine physics with metrics appearing as byproducts exist? It was the old idea of Ne'eman, Hehl, Sardanashvilly, and others 
[1]-[3] in $\mathrm{GL}(4, \mathbf{R})$-gauge approaches to gravitation. In some of our earlier papers we presented an alternative tetrad model of gravitation based on the global group GL(4, R) of internal symmetries $[4,5,6]$. Do realistic classical and quantum models ruled by affine groups exist? In typical theories the affine group occurs but in a rather different context. In nuclear matter theory it is used as a "non-invariance group". It rules kinematics but does not preserve dynamics. Instead of this, its generators satisfy some system of commutation relations with Hamiltonian, and essential information concerning the energy levels may be obtained on the basis of some ladder procedure. There exist also classical and quantum models of affinely-rigid body with kinematics ruled by the affine group, but with dynamics violating this symmetry and compatible at most with the Euclidean subgroup. Without potential, on the purely geodetic level, such models are non-physical, because they predict the unlimited contraction or expansion of the body. They are also deprived of the aesthetic beauty of invariant geodetic systems on Lie groups [7]; the possibility of rigorous analytical solution is also lost. Obviously, with appropriate potential terms, such models are applicable in a wide range of physical phenomena like nuclear and molecular dynamics, macroscopic elasticity, molecular crystals, micro-structured continua, and even astrophysical objects $[8,9]$. Nevertheless, at least from the academic point of view, it is an interesting idea to replace such models by affinely invariant geodetic models, metricallyrigid bodies by affinely-rigid ones, and spin by affine spin (hypermomentum) generating affine transformation centered at the center of mass [1, 10, 11, 12]. Some suggestions are presented below. It is interesting that replacing (pseudo)Euclidean and (pseudo)unitary symmetries by the affine one, one obtains essential (non-perturbative) nonlinearity, e.g., the generalized Born-Infeld-type nonlinearity in field theory [13].

\section{Invariant geodetic models}

When some reference configuration and Cartesian coordinates are fixed, the configuration space of the affinely-rigid body may be identified with the semi-direct product $\mathrm{GL}^{+}(n, \mathbf{R}) \times_{s}$ $\mathbf{R}^{n}$ (or simply with $\mathrm{GL}^{+}(n, \mathbf{R})$ when translational degrees of freedom are neglected). This means that the Lagrange (material, reference) coordinates $a^{K}$ and the Euler (spatial, current) variables $y^{i}$ are interrelated as follows: $y^{i}(t)=\varphi_{K}^{i}(t) a^{K}+x^{i}(t)$, where $x^{i}$ denotes the center of mass coordinates, and $\varphi^{i}{ }_{K}$ are generalized coordinates of internal motion (rotations and deformations). Spatial and material actions of $\mathrm{GL}^{+}(n, \mathbf{R})$ are given respectively by left and right regular translations: $\varphi \mapsto L \varphi, \varphi \mapsto \varphi R$. Euler and Lagrange additive translations act trivially on $x^{i}$ and $a^{K}$. Laboratory and material affine velocities are Liealgebraic objects defined respectively as follows: $\Omega=\dot{\varphi} \varphi^{-1}, \hat{\Omega}=\varphi^{-1} \dot{\varphi}=\varphi^{-1} \Omega \varphi$. The left and right regular translations affect them according to the rule: $L: \Omega \mapsto L \Omega L^{-1}, \hat{\Omega} \mapsto \hat{\Omega}$, $R: \Omega \mapsto \Omega, \hat{\Omega} \mapsto R^{-1} \hat{\Omega} R$. The co-moving representation of translational velocity is given by $\hat{v}^{A}=\left(\varphi^{-1}\right)^{A}{ }_{i} v^{i}=\left(\varphi^{-1}\right)_{i}^{A} \dot{x}^{i}$.

Kinetic energy (inertial metric tensor) is postulated in the additive form $T=T_{\mathrm{tr}}+$ $T_{\text {int }}$ (translational and internal parts). Left (spatially) invariant expressions for $T_{\text {int }}$ are constant-coefficients quadratic forms of $\hat{\Omega}$. Right (materially) invariant ones are built in a similar way of $\Omega$. The doubly invariant $T_{\text {int }}$ are combined of second-order Casimirs:

$$
T_{\text {int }}^{\text {aff }- \text { aff }}:=\frac{A}{2} \operatorname{Tr}\left(\Omega^{2}\right)+\frac{B}{2}(\operatorname{Tr} \Omega)^{2}=\frac{A}{2} \operatorname{Tr}\left(\hat{\Omega}^{2}\right)+\frac{B}{2}(\operatorname{Tr} \hat{\Omega})^{2},
$$


where $A, B$ are constants. Due to the semi-simplicity and non-compactness of $\operatorname{SL}(n, \mathbf{R})$, $T$ is not positively definite, however, this does not exclude its physical usefulness. It is non-degenerate when $A+n B \neq 0$. The special case $A=2 n, B=-2$ corresponds just to the standard normalization of the Killing metric; its degeneracy is due to the non-semisimplicity of $\mathrm{GL}(n, \mathbf{R})$.

Translational kinetic energies $T_{\text {tr }}$ are never doubly invariant. The highest possible symmetries for mathematically reasonable models are those affine in the space and Euclidean in the material, or conversely. Thus, these two possibilities can by written in the combined form:

$$
\left\{\begin{array}{l}
T_{\mathrm{tr}}^{\mathrm{met}-\mathrm{aff}} \\
T_{\mathrm{tr}}^{\mathrm{aff}-\mathrm{met}}
\end{array}\right\}=\frac{m}{2}\left\{\begin{array}{c}
g_{i j} \\
C_{i j}
\end{array}\right\} \dot{x}^{i} \dot{x}^{j}=\frac{m}{2}\left\{\begin{array}{c}
G_{A B} \\
\delta_{A B}
\end{array}\right\} \hat{v}^{A} \hat{v}^{B},
$$

where $g$ is the spatial metric tensor ( $\delta$ in the orthonormal basis), and $G, C$ are respectively the Green and Cauchy deformation tensors, $G_{A B}=g_{i j} \varphi^{i}{ }_{A} \varphi^{j}{ }_{B}, C_{i j}=\delta_{A B}\left(\varphi^{-1}\right)^{A}{ }_{i}\left(\varphi^{-1}\right)^{B}{ }_{j}$. Any left and right invariant twice covariant field on the affine group is degenerate, thus, non-applicable as a kinetic energy model. The above-quoted models of $T_{\text {tr }}$ show the highest reasonable invariance and fix our attention on the models of $T_{\text {int }}$ with the same symmetry properties, i.e., metric-affine and affine-metric:

$$
\begin{aligned}
\left\{\begin{array}{l}
T_{\text {int }}^{\text {met-aff }} \\
T_{\text {int }}^{\text {aff }}-\text { met }
\end{array}\right\} & =\frac{I}{2} \operatorname{Tr}\left\{\begin{array}{c}
\Omega^{T} \Omega \\
\hat{\Omega}^{T} \hat{\Omega}
\end{array}\right\}+T_{\text {int }}^{\text {aff }- \text { aff }} \\
& =\frac{I}{2} \operatorname{Tr}\left\{\begin{array}{c}
\Omega^{T} \Omega \\
\hat{\Omega}^{T} \hat{\Omega}
\end{array}\right\}+\frac{A}{2} \operatorname{Tr}\left\{\begin{array}{c}
\Omega^{2} \\
\hat{\Omega}^{2}
\end{array}\right\}+\frac{B}{2}\left(\operatorname{Tr}\left\{\begin{array}{c}
\Omega \\
\hat{\Omega}
\end{array}\right\}\right)^{2} .
\end{aligned}
$$

In some open domain of inertial constants $(I, A, B) \in \mathbf{R}^{3}$ the above expressions are positively definite, thus, "good" kinetic energies. The usual kinetic energy compatible with the d'Alembert principle has the following form: $T=T_{\mathrm{tr}}+T_{\mathrm{int}}=(m / 2) g_{i j} \dot{x}^{i} \dot{x}^{j}+$ $(1 / 2) g_{i j} \dot{\varphi}_{A}^{i} \dot{\varphi}_{B}^{j} J^{A B}$, where the inertial parameters are not primary ones but obtained from the measure $\mu$ describing the co-moving mass distribution: $m=\int d \mu(a), J^{A B}=$ $\int a^{A} a^{B} d \mu(a), \int a^{A} d \mu(a)=0$ (the total mass and second-order moment of its distribution; $J^{A B}=I \delta^{A B}$ in the isotropic case). Without an appropriate potential term the above $T$ is non-viable as a realistic Lagrangian, because the generic deformative motion would be non-bounded and passing through singularities. Unlike this, it was shown that geodetic models (2.1), (2.3) based on curved inertial metrics predict an open family of bounded and an open family of non-bounded orbits on $\mathrm{SL}(n, \mathbf{R})$. It is only dilatational motion that needs extra stabilization.

From now on, let us neglect translational motion. Legendre transformation may be expressed in any of the following convenient forms: $\Sigma^{i}{ }_{j}=\partial T_{\text {int }} / \partial \Omega^{j}{ }_{i}, \hat{\Sigma}^{A}{ }_{B}=\partial T_{\text {int }} / \partial \hat{\Omega}^{B}{ }_{A}$. The laboratory and co-moving affine spins $\Sigma, \hat{\Sigma}$ are respectively Hamiltonian generators (momentum mappings) of the left and right regular translations on $\operatorname{GL}(n, \mathbf{R})$. Geodetic Hamiltonians corresponding to (2.1), (2.3) have the following form:

$$
\begin{gathered}
\mathcal{T}_{\text {int }}^{\text {aff-aff }}=\frac{1}{2 A} \operatorname{Tr}\left\{\begin{array}{c}
\Sigma^{2} \\
\hat{\Sigma}^{2}
\end{array}\right\}-\frac{B}{2 A(A+n B)}\left(\operatorname{Tr}\left\{\begin{array}{c}
\Sigma \\
\hat{\Sigma}
\end{array}\right\}\right)^{2}, \\
\left\{\begin{array}{c}
\mathcal{T}_{\text {int }}^{\text {met-aff }} \\
\mathcal{T}_{\text {int }}^{\text {aff }} \text { met }
\end{array}\right\}=\frac{1}{2 \widetilde{I}} \operatorname{Tr}\left\{\begin{array}{c}
\Sigma^{T} \Sigma \\
\hat{\Sigma}^{T} \hat{\Sigma}
\end{array}\right\}+\frac{1}{2 \widetilde{A}} \operatorname{Tr}\left\{\begin{array}{c}
\Sigma^{2} \\
\hat{\Sigma}^{2}
\end{array}\right\}+\frac{1}{2 \widetilde{B}}\left(\operatorname{Tr}\left\{\begin{array}{c}
\Sigma \\
\hat{\Sigma}
\end{array}\right\}\right)^{2},
\end{gathered}
$$


where $\widetilde{I}=\left(I^{2}-A^{2}\right) / I, \widetilde{A}=\left(A^{2}-I^{2}\right) / A, \widetilde{B}=-(I+A)(I+A+n B) / B$. One can also use the following convenient representation:

$$
\left\{\begin{array}{c}
\mathcal{T}_{\text {it }}^{\text {met-aff }} \\
\mathcal{T}_{\text {int }}^{\text {aff }} \text { met }
\end{array}\right\}=\frac{C(2)}{2 \alpha}+\frac{C(1)^{2}}{2 \beta}+\frac{1}{2 \mu}\left\{\begin{array}{l}
\|S\|^{2} \\
\|V\|^{2}
\end{array}\right\},
$$

where $C(k)=\operatorname{Tr}\left(\Sigma^{k}\right)=\operatorname{Tr}\left(\hat{\Sigma}^{k}\right)$, and $S=\Sigma-\Sigma^{T}, V=\hat{\Sigma}-\hat{\Sigma}^{T}$ are spin and vorticity (generators of spatial and material rotations), $\|S\|$ and $\|V\|$ are their magnitudes, $\|S\|^{2}=$ $-(1 / 2) \operatorname{Tr}\left(S^{2}\right) \geq 0,\|V\|^{2}=-(1 / 2) \operatorname{Tr}\left(V^{2}\right) \geq 0$, and $\alpha, \beta, \mu$ are constants: $\alpha:=I+A$, $\beta:=-(I+A)(I+A+n B) / B, \mu:=\left(I^{2}-A^{2}\right) / I$. Obviously, $\mathcal{T}_{\text {int }}^{\text {aff-aff }}$ is obtained by putting $I=0$, i.e., $\mu=0$.

It is also convenient to introduce the auxiliary quantities $q \in \mathbf{R}, \omega, \hat{\omega} \in \operatorname{SL}(n, \mathbf{R})^{\prime}$, and the canonical momenta conjugate to them $p \in \mathbf{R}, \sigma, \hat{\sigma} \in \operatorname{SL}(n, \mathbf{R})^{\prime *} \simeq \operatorname{SL}(n, \mathbf{R})^{\prime}$, where $\operatorname{det} \varphi=\exp (n q), \omega=\Omega-(1 / n)(\operatorname{Tr} \Omega) I_{n}=\Omega-\dot{q} I_{n}, \hat{\omega}=\hat{\Omega}-(1 / n) \operatorname{Tr}(\hat{\Omega}) I_{n}=\hat{\Omega}-\dot{q} I_{n}$, $\sigma=\Sigma-(p / n) I_{n}, \hat{\sigma}=\hat{\Sigma}-(p / n) I_{n}$, and $I_{n}$ is the unit $n \times n$ matrix. This is the splitting of $\mathrm{GL}(n, \mathbf{R})$ into pure dilatational and isochoric $\mathrm{SL}(n, \mathbf{R})$ parts. One can easily show that:

$$
\begin{gathered}
\mathcal{T}_{\text {int }}^{\text {aff-aff }}=\frac{C_{\mathrm{SL}(n)}(2)}{2 A}+\frac{p^{2}}{2 n(A+n B)}, \\
\left\{\begin{array}{l}
\mathcal{T}_{\text {int }}^{\text {met-aff }} \\
\mathcal{T}_{\text {int }}^{\text {aff }- \text { met }}
\end{array}\right\}=\frac{C_{\mathrm{SL}(n)}(2)}{2(I+A)}+\frac{p^{2}}{2 n(I+A+n B)}+\frac{I}{2\left(I^{2}-A^{2}\right)}\left\{\begin{array}{l}
\|S\|^{2} \\
\|V\|^{2}
\end{array}\right\},
\end{gathered}
$$

where $C_{\mathrm{SL}(n)}(2)=\operatorname{Tr}\left(\sigma^{2}\right)=\operatorname{Tr}\left(\hat{\sigma}^{2}\right)$. It is seen that $\mathcal{T}_{\text {int }}^{\text {met-aff }}$ and $\mathcal{T}_{\text {int }}^{\text {aff-met }}$ differ merely by geometrically interesting $\|S\|^{2}$ - and $\|V\|^{2}$-correction terms from the doubly invariant $\mathcal{T}_{\text {int }}^{\text {aff }- \text { aff }}$ in which $A$ is to be replaced by $(I+A)$.

As mentioned, on the incompressible $\operatorname{SL}(n, \mathbf{R})$ level, the above geodetic models predict bounded elastic vibrations and may be analytically investigated due to their invariance under regular translations. When the total $\operatorname{GL}(n, \mathbf{R})$ is admitted, dilatations must be stabilized by some term $\mathcal{V}(q)$, e.g., of the oscillator form $\mathcal{V}=(\kappa / 2) q^{2}$, or the narrow and deep potential well around $q=0$.

Classical equations may be analyzed and solved with the use of Poisson brackets and exponential mapping.

\section{Quantization ideas}

Integration elements corresponding to Haar measures $\alpha, \lambda$ on the affine and linear groups are given respectively as follows: $d \alpha(x, \varphi)=(\operatorname{det} \varphi)^{-n-1} d x^{1} \ldots d x^{n} d \varphi^{1}{ }_{1} \ldots d \varphi^{n}{ }_{n}, d \lambda(\varphi)=$ $(\operatorname{det} \varphi)^{-n} d \varphi^{1}{ }_{1} \ldots d \varphi^{n}{ }_{n}$. Quantum mechanics of the affinely-rigid body is formulated in Hilbert spaces $\mathrm{L}^{2}\left(\mathrm{GL}(n, \mathbf{R}) \times{ }_{s} \mathbf{R}^{n}, \alpha\right)$ and $\mathrm{L}^{2}(\mathrm{GL}(n, \mathbf{R}), \lambda)$ respectively for systems with and without translational degrees of freedom. Kinetic energy operator $\mathbf{T}$ has the standard form $\mathbf{T}=\left(\hbar^{2} / 2\right) \Delta$, where $\Delta$ is the Laplace-Beltrami operator based on the metric tensor underlying the classical kinetic energy, $\Delta=g^{\mu \nu} \nabla_{\mu} \nabla_{\nu}$. The direct computation of $\Delta$ is rather complicated and the resulting formula is completely non-readable. However, the group structure enables one to express $\mathbf{T}$ in terms of differential operators $\boldsymbol{\Sigma}_{j}^{i}=-i \hbar \varphi_{A}^{i}\left(\partial / \partial \varphi_{A}{ }_{j}\right), \hat{\boldsymbol{\Sigma}}^{A}{ }_{B}=-i \hbar \varphi_{B}^{i}\left(\partial / \partial \varphi_{A}^{i}\right)$ generating left and right regular translations. They are operators of laboratory and co-moving components of the affine spin. 
Regular translations are unitary in the sense of scalar product based on the Haar measure. Therefore, $\boldsymbol{\Sigma}, \hat{\boldsymbol{\Sigma}}$ are formally self-adjoint. Of course, being differential operators, they are unbounded, thus, they are not Hermitian in the literal mathematical sense. Nevertheless, they are good physical observables.

Finally, kinetic energy operators have the following form:

$$
\begin{aligned}
\mathcal{T}_{\text {int }}^{\text {aff-aff }} & =\frac{1}{2 A}\left\{\begin{array}{c}
\boldsymbol{\Sigma}_{i}^{i}{ }_{j} \boldsymbol{\Sigma}^{j}{ }_{i} \\
\hat{\boldsymbol{\Sigma}}^{A}{ }_{B} \hat{\boldsymbol{\Sigma}}^{B}{ }_{A}
\end{array}\right\}-\frac{B}{2 A(A+n B)}\left\{\begin{array}{c}
\boldsymbol{\Sigma}_{i}^{i} \boldsymbol{\Sigma}^{j}{ }_{j} \\
\hat{\boldsymbol{\Sigma}}^{A}{ }_{A} \hat{\boldsymbol{\Sigma}}^{B}{ }_{B}
\end{array}\right\}, \\
\left\{\begin{array}{c}
\mathcal{T}_{\text {int }}^{\text {met-aff }} \\
\mathcal{T}_{\text {int }}^{\text {aff }} \text { met }
\end{array}\right\} & =\frac{1}{2 \widetilde{I}}\left\{\begin{array}{c}
g_{i k} g^{j l} \boldsymbol{\Sigma}_{j}^{i} \boldsymbol{\Sigma}^{k}{ }_{l} \\
\delta_{A B} \delta^{C D} \hat{\boldsymbol{\Sigma}}^{A}{ }_{C} \hat{\boldsymbol{\Sigma}}^{B}{ }_{D}
\end{array}\right\} \\
& +\frac{1}{2 \widetilde{A}}\left\{\begin{array}{c}
\boldsymbol{\Sigma}^{i}{ }_{j} \boldsymbol{\Sigma}^{j}{ }_{i} \\
\hat{\boldsymbol{\Sigma}}^{A}{ }_{B} \hat{\boldsymbol{\Sigma}}^{B}{ }_{A}
\end{array}\right\}+\frac{1}{2 \widetilde{B}}\left\{\begin{array}{c}
\boldsymbol{\Sigma}_{i}^{i}{ }_{i} \boldsymbol{\Sigma}_{j}^{j} \\
\hat{\boldsymbol{\Sigma}}^{A}{ }_{A} \hat{\boldsymbol{\Sigma}}^{B}{ }_{B}
\end{array}\right\} .
\end{aligned}
$$

Representations (2.6), (2.7), (2.8) are also possible; the meaning of the operator symbols is obvious from the later formulas, and the quantized dilatational momentum is given by $\mathbf{p}=-i \hbar(\partial / \partial q)$.

The above kinetic energies restricted to $\mathrm{SL}(n, \mathbf{R})$ have both discrete and continuous spectra. On $\operatorname{GL}(n, \mathbf{R})$, just as in the classical case, one has to use stabilizing dilatational potential $\mathcal{V}(q)$ to prevent plane-wave dilatational solutions. As previously, harmonic oscillator and potential well are the simplest and most convincing models, at least in nuclear physics.

There is a very important point concerning the quantized model. Namely, it belongs to the very fundamentals of the wave version of quantum mechanics that the wave function has to be one-valued (and satisfy other regularity requirements). Although, it is wellmotivated in $\mathbf{R}^{n}$, but it is not necessarily so in multiply connected manifolds with finite homotopy groups. Then one can use the universal covering manifold of the configuration space, in our case simply the universal covering group $\overline{\mathrm{GL}(n, \mathbf{R})}$, as a proper, so to speak "hidden", configuration space. To preserve the usual statistical interpretation, we must only demand the probability density $\bar{\Psi} \Psi$ to be one-valued, thus, projectable from the covering manifold to the original configuration space. Nevertheless, the class of acceptable wave functions $\Psi$ is essentially wider, they may be non-projectable, i.e., multi-valued from the point of view of the original configuration space. That is the fact, e.g., in rigid body mechanics, where for $n \geq 3$ the configuration space $\mathrm{SO}(n, \mathbf{R})$ is doubly-connected and universally covered by the group $\operatorname{Spin}(n)$, i.e., $\mathrm{SU}(2)$ in the physical case $n=3$. Therefore, the two-valued wave functions possessing two different signs at the same $\mathrm{SO}(3, \mathbf{R})$ point are admitted [14]. Obviously, they are single-valued on SU(2). According to the Peter-Weyl theorem, the wave functions on $\mathrm{SU}(2)$ may be expanded with respect to matrix elements of unitary irreducible representations $D_{m m^{\prime}}^{s}$. For half-integer and integer values of $s$ these functions satisfy respectively $D_{m m^{\prime}}^{s}(-u)=\mp D_{m m^{\prime}}^{s}(u)$ for any $u \in \mathrm{SU}(2)$; obviously $\pm u$ project onto the same element of $\mathrm{SO}(3, \mathbf{R})$. But $\bar{\Psi} \Psi$ must be one-valued, therefore, a kind of superselection rule appears according to which states with half-integer and integer $s$ cannot be superposed with each other. This is a toy model of the fermionic and bosonic sectors.

The same problem appears in quantum mechanics of affinely-rigid body. Indeed, for $n \geq 3 \mathrm{GL}^{+}(n, \mathbf{R})$ and $\mathrm{SL}(n, \mathbf{R})$ are doubly-connected. It is an interesting and intriguing fact that the covering groups $\overline{\mathrm{GL}^{+}(n, \mathbf{R})}$ and $\overline{\mathrm{SL}(n, \mathbf{R})}$ are nonlinear, i.e., do not possess 
faithful realizations through finite matrices. Their description as manifolds may be based on the polar splitting $\varphi=U A=B U=\left(U A U^{-1}\right) U$, where $U \in \operatorname{SO}(n, \mathbf{R})$, and $A, B \in$ $\operatorname{Symm}^{+}(n, \mathbf{R})$ are symmetric and positively definite. The splittings are unique, and in this way $\mathrm{GL}^{+}(n, \mathbf{R})$ as a manifold may be identified with the Cartesian product $\mathrm{SO}(n, \mathbf{R}) \times$ $\operatorname{Symm}^{+}(n, \mathbf{R})$ or $\operatorname{Symm}^{+}(n, \mathbf{R}) \times \mathrm{SO}(n, \mathbf{R}) \cdot \operatorname{Symm}^{+}(n, \mathbf{R})$ is topologically $\mathbf{R}^{n(n+1) / 2}\left(\mathbf{R}^{6}\right.$ in the physical three-dimensional case). Therefore, the covering manifold may be identified with $\operatorname{Spin}(n) \times \operatorname{Symm}^{+}(n, \mathbf{R})$ or $\operatorname{Symm}^{+}(n, \mathbf{R}) \times \operatorname{Spin}(n)$. Wave functions may be expanded as previously: $\Psi(u, A)=\sum C_{m m^{\prime}}^{s}(A) D_{m m^{\prime}}^{s}(u)$, with the same restriction that only half-integer or integer $s$ may appear in a given expansion.

In our highly-symmetric models it is more convenient to use the two-polar decomposition. It consists of diagonalization of $A$ or $B$, e.g., $A=R D R^{-1}, R \in \operatorname{SO}(n, \mathbf{R})$, and $D \in$ $\operatorname{Diag}\left(\mathbf{R}^{n}\right)$ is diagonal. Assigning $L:=U R \in \mathrm{SO}(n, \mathbf{R})$, we have finally: $\varphi=L D R^{-1}$. It is also convenient to denote $D_{i i}=Q^{i}=\exp \left(q^{i}\right)$. The previously introduced dilatational variable $q$ is the "centre of mass" of $q^{i}$ "s, i.e., $q=\left(q^{1}+\ldots+q^{n}\right) / n$, and $p=p_{1}+\ldots+p_{n}$, where $p_{i}$ are canonical momenta conjugate to $q^{i}$ (those conjugate to $Q^{i}$ will be denoted by $P_{i}$ ). In this way $\varphi \in \mathrm{GL}^{+}(n, \mathbf{R})$ is identified with a triplet $(L ; D(q) ; R) \in \mathrm{SO}(n, \mathbf{R}) \times \mathbf{R}^{n} \times \mathrm{SO}(n, \mathbf{R})$. This splitting is not unique. Let us describe this non-uniqueness explicitly, because it is essential for taking into account half-integer angular momentum of extended deformable bodies.

Let $K \in \mathrm{O}(n, \mathbf{R})$ denote the finite group of orthogonal matrices which have exactly one non-vanishing entry in every row and column; obviously, these entries equal to \pm 1 . The subgroup $K^{+}:=K \cap \mathrm{SO}(n, \mathbf{R})$ consists of afore-defined matrices with the determinants equal to +1 . It is easy to see that the groups $K, K^{+}$have respectively $(2 n) n$ ! and $(n) n$ ! elements. For any $U \in K$, the similarity transformation $\operatorname{Diag}\left(\mathbf{R}^{n}\right) \ni D \mapsto U^{-1} D U \in \operatorname{Diag}\left(\mathbf{R}^{n}\right)$ results in a permutation of diagonal elements of $D:\left(Q^{1}, \ldots, Q^{n}\right) \mapsto\left(Q^{\pi_{U}(1}, \ldots, Q^{n)}\right)$, i.e., $\left(q^{1}, \ldots, q^{n}\right) \mapsto\left(q^{\pi_{U}(1}, \ldots, q^{n)}\right)$. The assignment $K \ni U \mapsto \pi_{U} \in \mathrm{S}^{(n)}$ is a $(2 n: 1)$ epimorphism of $K$ onto the permutation group $\mathrm{S}^{(n)}$. Restricting it to $K^{+}$, we obtain an ( $n: 1)$-epimorphism.

Let $\mathrm{GL}^{+(n)}(n, \mathbf{R})$ denote the subset of $\mathrm{GL}^{+}(n, \mathbf{R})$ with the simple spectra of deformation tensors, and $M^{(n)}$ be the corresponding subset of $\mathrm{SO}(n, \mathbf{R}) \times \mathbf{R}^{n} \times \mathrm{SO}(n, \mathbf{R})$ consisting of such triplets $\left(L ; q^{1}, \ldots, q^{n} ; R\right)$ that all $q^{i}$ 's are pairwise disjoint. Let $K^{+}$act on $M^{(n)}$ according to the rule: $\left(L ; q^{1}, \ldots, q^{n} ; R\right) \mapsto\left(L U ; q^{\pi_{U}(1}, \ldots, q^{n)} ; R U\right)$. Let us denote the corresponding transformation group of $M^{(n)}$ by $H^{(n)}$. It is clear that $\mathrm{GL}^{+(n)}(n, \mathbf{R})$ is diffeomorphic with $M^{(n)} / H^{(n)}$, i.e., $\mathrm{GL}^{+(n)}(n, \mathbf{R}) \simeq M^{(n)} / H^{(n)}$. Of course, this situation of non-degenerate spectra is a generic one, so this is the main part of the multi-valuedness of the two-polar decomposition.

To describe the covering group $\overline{\mathrm{GL}^{+}(n, \mathbf{R})}$ one should use the following auxiliary manifold: $\operatorname{Spin}(n) \times \mathbf{R}^{n} \times \operatorname{Spin}(n)\left(\mathrm{SU}(2) \times \mathbf{R}^{3} \times \mathrm{SU}(2)\right.$ for $\left.n=3\right)$. Let $\tau: \operatorname{Spin}(n) \rightarrow$ $\mathrm{SO}(n, \mathbf{R})$ denote the canonical projection; $\overline{K^{+}} \subset \operatorname{Spin}(n)$ denotes the $(2 n) n$ !-element subgroup $\tau^{-1}\left(K^{+}\right)$. The manifold $M^{(n)}$ introduced above is covered by $\overline{M^{(n)}}$, i.e., the subset of such triplets $\left(l ; q^{1}, \ldots, q^{n} ; r\right) \in \operatorname{Spin}(n) \times \mathbf{R}^{n} \times \operatorname{Spin}(n)$ that all $q^{i}$ 's are pairwise disjoint. And $\overline{K^{+}}$induces on $\overline{M^{(n)}}$ the transformation group $\overline{H^{(n)}}$ the action of which is given by the following rule: $\left(l ; q^{1}, \ldots, q^{n} ; r\right) \mapsto\left(l u ; q^{\pi_{\tau(u)}(1}, \ldots, q^{n)} ; r u\right)$, where $u \in \operatorname{Spin}(n)(\operatorname{SU}(2)$ if $n=3)$. The corresponding generic part of the configuration space is given by the quotient manifold $Q^{(n)} \simeq \overline{M^{(n)}} / \overline{H^{(n)}}$. In situations when there are coincidences of $q^{i}$ s, i.e., when 
the spectra of deformations tensors are degenerate, description is more complicated. Such configurations are non-generic, and they need some special treatment. It is impossible to present all details here, so we will only describe a brief scheme for this.

Let $M^{\left(k ; p_{1} \ldots p_{n}\right)}$ be the set of such triplets $\left(L ; q^{1}, \ldots, q^{n} ; R\right)$ that there are only $k$ different $q^{i}$ s, every one with a multiplicity factor $p_{\sigma}, \sum_{\sigma=1}^{k} p_{\sigma}=n$. Let us take the transformation group $H^{\left(k ; p_{1} \ldots p_{n}\right)}$ with the following action on $M^{\left(k ; p_{1} \ldots p_{n}\right)}:\left(L ; q^{1}, \ldots, q^{n} ; R\right) \mapsto$ $\left(L U ; q^{\pi_{U}(1}, \ldots, q^{n)} ; R U\right)$, where $U$ runs over the group generated by $K$ and the subgroup $W^{\left(k ; p_{1} \ldots p_{n}\right)} \subset \mathrm{SO}(n, \mathbf{R})$ composed of $k p_{\sigma} \times p_{\sigma}$ blocks, every one in the corresponding $\mathrm{SO}\left(p_{\sigma}, \mathbf{R}\right)$. The generic subset $Q^{\left(k ; p_{1} \ldots p_{n}\right)}$ is given by the quotient $M^{\left(k ; p_{1} \ldots p_{n}\right)} / H^{\left(k ; p_{1} \ldots p_{n}\right)}$. When the half-integer spin is to be taken into account, we must consider the manifold $\overline{M^{\left(k ; p_{1} \ldots p_{n}\right)}}$ consisting of triplets $\left(l ; q^{1}, \ldots, q^{n} ; r\right)$, where $l, r \in \operatorname{Spin}(n)$, and $\left(q^{1}, \ldots, q^{n}\right)$ are degenerate as above. Then $\overline{H^{\left(k ; p_{1} \ldots p_{n}\right)}}=\tau^{-1}\left(H^{\left(k ; p_{1} \ldots p_{n}\right)}\right) \subset \operatorname{Spin}(n, \mathbf{R})$, and the manifold of the corresponding degenerate configuration is the quotient $\overline{M^{\left(k ; p_{1} \ldots p_{n}\right)}} / \overline{H^{\left(k ; p_{1} \ldots p_{n}\right)}}$ taken with respect to the action $\left(l ; q^{1}, \ldots, q^{n} ; r\right) \mapsto\left(l u ; q^{\pi_{\tau(u)}(1}, \ldots, q^{n)} ; r u\right)$, where $u \in$ $\overline{H^{\left(k ; p_{1} \ldots p_{n}\right)}}$. When $k<n$, i.e., at least one multiplicity factor is nontrivial, the group $\overline{H^{\left(k ; p_{1} \ldots p_{n}\right)}}$ is continuous, and the resulting quotient is lower-dimensional. In the physical case $n=3$, we have obviously only two possibilities of nontrivial blocks, $\mathrm{SO}(2, \mathbf{R}) \times$ $\mathrm{SO}(1, \mathbf{R})$ and the total $\mathrm{SO}(3, \mathbf{R})$ (respectively two of $q$ 's or all of them equal); obviously, $\mathrm{SO}(1, \mathbf{R})=\{1\}$.

Let us now just concentrate on the physical case $n=3$. In our highly-symmetric geodetic models the projections of spin onto some space-fixed $z$-axis and the projection of vorticity onto some body-fixed $z^{\prime}$-axis are good quantum numbers. Our wave functions may be expanded as follows:

$$
\Psi(u, q, v)=\sum_{s, j} \sum_{m, n=-s}^{s} \sum_{k, l=-j}^{j} D_{m n}^{s}(u) f_{\left(\begin{array}{c}
n k \\
m l
\end{array}\right)}^{s j}(q) D_{k l}^{j}\left(v^{-1}\right),
$$

where $u, v \in \mathrm{SU}(2)$, and $q$ is here an abbreviation for $\left(q^{1}, q^{2}, q^{3}\right),(m, n)$ and $(k, l)$ run over the range from $-s$ to $s$ and $-j$ to $j$ respectively in integer steps, whereas $s, j$ are nonnegative integers starting from 0 or positive half-integers starting from $1 / 2$. But, just as in rigid body mechanics, there is a superselection rule. Namely, if $|\Psi|$ is to be one-valued, then either $s, j$ must be simultaneously half-integer or simultaneously integer. The reduced invariant-dependent amplitudes $f^{s j}\left(q^{1}, q^{2}, q^{3}\right)$ vanish in the mixed case, i.e., if $(j-s)$ is half-integer. The case of degenerate triplets $\left(q^{1}, q^{2}, q^{3}\right)$ is too complicated to be described here in detail; the general rules follow from the above discussion. Namely, the $f$-amplitudes must be chosen in such a way as not to distinguish triplets $(u, q, v)$ equivalent in the above sense.

When there is no external potential, i.e., in purely geodetic models, it is convenient to restrict ourselves to expansions with fixed values of $s, j, m, l$ :

$$
\Psi_{m l}^{s j}(u, q, v)=\sum_{n=-s}^{s} \sum_{k=-j}^{j} D_{m n}^{s}(u) f_{n k}^{s j}(q) D_{k l}^{j}\left(v^{-1}\right) .
$$

Everything said above applies to $\mathrm{SL}(3, \mathbf{R})$ geodetic situations when dilatations are stabilized with the help of some potential $\mathcal{V}(q)$ (now $q=\left(q^{1}+q^{2}+q^{3}\right) / 3$ ), or even to more 
general non-geodetic situations when the potential energy is non-trivial but depends only on deformation invariants, $\mathcal{V}\left(q^{1}, q^{2}, q^{3}\right)$.

For fixed $s, j$ the reduced amplitude $f^{s j}$ is a $q^{i}$-dependent $(2 s+1) \times(2 j+1)$ matrix. It satisfies the family of reduced Schrödinger eigenequations for energy levels: $\mathbf{H}^{s j} f^{s j}=$ $E^{s j} f^{s j}$, where for our model $\mathcal{T}_{\text {int }}^{\text {aff-aff }}$ we have:

$$
\begin{aligned}
\mathbf{H}^{s j} f^{s j} & =-\frac{\hbar^{2}}{2 A} D f^{s j}+\frac{\hbar^{2} B}{2 A(A+n B)} \frac{\partial^{2}}{\partial q^{2}} f^{s j}+\frac{1}{32 A} \sum_{a, b} \frac{\left(\overleftarrow{S}_{a b}^{j}-\vec{S}_{a b}^{s}\right)^{2}}{\operatorname{sh}^{2}\left[\left(q^{a}-q^{b}\right) / 2\right]} f^{s j} \\
& -\frac{1}{32 A} \sum_{a, b} \frac{\left(\overleftarrow{S}_{a b}^{j}+\vec{S}_{a b}^{s}\right)^{2}}{\operatorname{ch}^{2}\left[\left(q^{a}-q^{b}\right) / 2\right]} f^{s j}+\mathcal{V} f^{s j}
\end{aligned}
$$

where $D=(1 / P) \sum_{a}\left(\partial / \partial q^{a}\right) P\left(\partial / \partial q^{a}\right), P=\prod_{a \neq b}\left|\operatorname{sh}\left(q^{a}-q^{b}\right)\right|, S_{a b}^{j}$ is the $(a, b)$-th $(\hbar$ included) matrix of the $j$-th angular momentum, i.e, rotations in the $(a, b)$-th plane of $\mathbf{R}^{n}$ (for $n=3, S_{a b}^{j}$ are $(2 j+1) \times(2 j+1)$-matrices), $\overleftarrow{S}_{a b}^{j} f^{s j}=f^{s j} S_{a b}^{j}, \vec{S}_{a b}^{s} f^{s j}=S_{a b}^{s} f^{s j}, \mathcal{V}$ denotes the dilatation-stabilizing potential. The structure of equations does not change when, besides of $q=\left(q^{1}+\ldots+q^{n}\right) / n, \mathcal{V}$ depends also on other deformation invariants (eigenvalues of deformation tensors).

The Haar measure in the two-polar representation is given by the following expression: $d \lambda(l, q, r)=\prod_{i \neq j}\left|\operatorname{sh}\left(q^{i}-q^{j}\right)\right| d \mu(l) d \mu(r) d q^{1} \ldots d q^{n}$, where $\mu$ denotes the unity-normalized Haar measure on $\mathrm{SO}(n, \mathbf{R})$.

Remark: sometimes it is convenient to use the modified deformation amplitude $g=\sqrt{P} f$. The resulting Schrödinger equation is analogous to (3.5) with the difference that $D$ is replaced by $\sum_{a}\left(\partial^{2} / \partial\left(q^{a}\right)^{2}\right)-(\hbar / 2 m) P^{-2}+\left(\hbar^{2} / 4 m\right) P^{-1} \sum_{a}\left(\partial P / \partial q^{a}\right)^{2}$, i.e., the usual $\mathbf{R}^{n_{-}}$ Laplacian modified by some extra "potential" term. The scalar product representation is then also modified in an appropriate way.

In three dimensions the effective Hamiltonians $\mathbf{H}^{s j}$ corresponding to $\mathcal{T}_{\text {int }}^{\text {net-aff }}, \mathcal{T}_{\text {int }}^{\text {aff }- \text { met }}$ are as follows:

$$
\begin{aligned}
\mathbf{H}^{s j} f^{s j} & =-\frac{\hbar^{2}}{2 \alpha} D f^{s j}-\frac{\hbar^{2}}{2 \beta} \frac{\partial^{2}}{\partial q^{2}} f^{s j}+\frac{1}{32 \alpha} \sum_{a, b} \frac{\left(\overleftarrow{S}_{a b}^{j}-\vec{S}_{a b}^{s}\right)^{2}}{\operatorname{sh}^{2}\left[\left(q^{a}-q^{b}\right) / 2\right]} f^{s j} \\
& -\frac{1}{32 \alpha} \sum_{a, b} \frac{\left(\overleftarrow{S}_{a b}^{j}+\vec{S}_{a b}^{s}\right)^{2}}{\operatorname{ch}^{2}\left[\left(q^{a}-q^{b}\right) / 2\right]} f^{s j}+\frac{\hbar^{2}}{2 \mu}\left\{\begin{array}{c}
s(s+1) \\
j(j+1)
\end{array}\right\} f^{s j}+\mathcal{V} f^{s j}
\end{aligned}
$$

For a general $n$ the above $\hbar^{2} s(s+1)$ and $\hbar^{2} j(j+1)$ terms are replaced by the corresponding eigenvalues of the second-order Casimirs of $\mathrm{SO}(n, \mathbf{R})$. The terms with denominators $\operatorname{ch}^{2}\left[\left(q^{a}-q^{b}\right) / 2\right]$ and $\operatorname{sh}^{2}\left[\left(q^{a}-q^{b}\right) / 2\right]$ describe respectively the effective attraction (acting even without any $\mathcal{V})$ and repulsion. For the scalar situation, when $s=j=0$, there are only terms $-\left(\hbar^{2} / 2 \alpha\right) D f^{00}-\left(\hbar^{2} / 2 \beta\right)\left(\partial^{2} f^{00} / \partial q^{2}\right)+\mathcal{V} f^{00}$ in the Schrödinger operator. The special case $s=j=1 / 2$ is very interesting, because $S_{a b}^{1 / 2}=(\hbar / 2) \sigma_{a b}$, i.e., spin and vorticity are represented by Pauli matrices multiplied by $\hbar / 2$. Obviously, in two dimensions, when the covering kernel is isomorphic with $\mathbf{Z}$, there is no half-integer angular momentum.

Let us recall that in the exceptional case $n=3$ the bi-indices $(a, b)$ may be replaced by the dual indices $c$, where $c \neq a, b$, namely, $S_{a}^{j}=(1 / 2) \epsilon_{a b c} S_{b c}^{j}, S_{a b}^{j}=\epsilon_{a b c} S_{c}^{j}$. Then after 
some calculations it may be shown that two terms of $\mathbf{H}^{s j}$ controlled by the factor $1 / 32 \alpha$ has the following explicit form:

$$
\begin{aligned}
& \frac{\left(S_{1}^{s}\right)^{2} f^{s j}-2 S_{1}^{s} f^{s j} S_{1}^{j}+f^{s j}\left(S_{1}^{j}\right)^{2}}{16 \alpha \operatorname{sh}^{2}\left[\left(q^{2}-q^{3}\right) / 2\right]}+\frac{\left(S_{2}^{s}\right)^{2} f^{s j}-2 S_{2}^{s} f^{s j} S_{2}^{j}+f^{s j}\left(S_{2}^{j}\right)^{2}}{16 \alpha \operatorname{sh}^{2}\left[\left(q^{1}-q^{3}\right) / 2\right]} \\
& +\frac{\left(S_{3}^{s}\right)^{2} f^{s j}-2 S_{3}^{s} f^{s j} S_{3}^{j}+f^{s j}\left(S_{3}^{j}\right)^{2}}{16 \alpha \operatorname{sh}^{2}\left[\left(q^{1}-q^{2}\right) / 2\right]}-\frac{\left(S_{1}^{s}\right)^{2} f^{s j}+2 S_{1}^{s} f^{s j} S_{1}^{j}+f^{s j}\left(S_{1}^{j}\right)^{2}}{16 \alpha \operatorname{ch}^{2}\left[\left(q^{2}-q^{3}\right) / 2\right]} \\
& -\frac{\left(S_{2}^{s}\right)^{2} f^{s j}+2 S_{2}^{s} f^{s j} S_{2}^{j}+f^{s j}\left(S_{2}^{j}\right)^{2}}{16 \alpha \operatorname{ch}^{2}\left[\left(q^{1}-q^{3}\right) / 2\right]}-\frac{\left(S_{3}^{s}\right)^{2} f^{s j}+2 S_{3}^{s} f^{s j} S_{3}^{j}+f^{s j}\left(S_{3}^{j}\right)^{2}}{16 \alpha \operatorname{ch}^{2}\left[\left(q^{1}-q^{2}\right) / 2\right]} .
\end{aligned}
$$

Depending on the relationship between $s$ and $j$, the $\operatorname{SL}(3, \mathbf{R})$-geodetic spectrum is discrete (bounded states) or continuous. The same is true for the total GL(3, R)-dynamics, when an appropriate dilatation-stabilizing potential is used. Standard terms $\left(\hbar^{2} / 2 \mu\right) s(s+1)$ and $\left(\hbar^{2} / 2 \mu\right) j(j+1)$ appearing respectively in the metric-affine and affine-metric models as some corrections to the standard affine-affine spectrum are very interesting. They seem to be confirmed by experimental data concerning nuclear and hadronic energetic (mass) spectra. The controlling quantum numbers $s, j$ have to do with spin and probably isospin properties. As it was mentioned before, the third and fourth terms of (3.6) simply vanish for the scalar case, when $s=j=0$. In the physical dimension $n=3$, the spinor-spinor case $s=j=1 / 2$ is also remarkably simpler than the general situation, because we have $S_{a}^{1 / 2}=(\hbar / 2) \sigma_{a}$ and $\left[S_{a}^{1 / 2}\right]^{2}=\left(\hbar^{2} / 4\right) I_{2}$, where $I_{2}$ is the unit $2 \times 2$ matrix.

Finally, let us mention the planar case $n=2$, which is also of some physical interest. Then in the two-polar decomposition we have:

$$
\begin{aligned}
& L=\left[\begin{array}{cc}
\cos \alpha & -\sin \alpha \\
\sin \alpha & \cos \alpha
\end{array}\right], \quad R=\left[\begin{array}{cc}
\cos \beta & -\sin \beta \\
\sin \beta & \cos \beta
\end{array}\right], \\
& S=p_{\alpha}\left[\begin{array}{cc}
0 & -1 \\
1 & 0
\end{array}\right], \quad V=p_{\beta}\left[\begin{array}{cc}
0 & -1 \\
1 & 0
\end{array}\right],
\end{aligned}
$$

where $p_{\alpha}, p_{\beta}$ are canonical momenta conjugate to $\alpha, \beta$. It is convenient to introduce new variables $q:=\left(q^{1}+q^{2}\right) / 2$ and $x:=q^{2}-q^{1}$, their conjugate momenta are respectively $p=$ $p_{1}+p_{2}$ and $y=\left(p_{2}-p_{1}\right) / 2$. The Haar measure on $\operatorname{GL}(2, \mathbf{R})$ is given by $d \lambda\left(\alpha ; q^{1}, q^{2} ; \beta\right)=$ $\left|\operatorname{sh}\left(q^{1}-q^{2}\right)\right| d \alpha d \beta d q^{1} d q^{2}$, i.e., $d \lambda(\alpha ; q, x ; \beta)=|\operatorname{sh} x| d \alpha d \beta d x d q$. The Fourier expansion of wave functions with respect to $\alpha, \beta$ is given by $\Psi(\alpha ; q, x ; \beta)=\sum_{m, n \in \mathbf{Z}} f^{m n}(q, x) e^{i m \alpha} e^{i n \beta}$. The reduced Hamiltonians corresponding to $\mathcal{T}_{\text {int }}^{\text {aff }- \text { aff }}, \mathcal{T}_{\text {int }}^{\text {met-aff }}, \mathcal{T}_{\text {int }}^{\text {aff-met }}$ are as follows:

$$
\begin{aligned}
\mathbf{H}^{m n} f^{m n} & =-\frac{\hbar^{2}}{2 A} D f^{m n}+\frac{\hbar^{2} B}{2 A(A+2 B)} \frac{\partial^{2}}{\partial q^{2}} f^{m n} \\
& +\frac{\hbar^{2}(n-m)^{2}}{16 A \operatorname{sh}^{2}(x / 2)} f^{m n}-\frac{\hbar^{2}(n+m)^{2}}{16 A \operatorname{ch}^{2}(x / 2)} f^{m n}+\mathcal{V} f^{m n} \\
\mathbf{H}^{m n} f^{m n} & =-\frac{\hbar^{2}}{2 \alpha} D f^{m n}-\frac{\hbar^{2}}{2 \beta} \frac{\partial^{2}}{\partial q^{2}} f^{m n}+\frac{\hbar^{2}(n-m)^{2}}{16 \alpha \operatorname{sh}^{2}(x / 2)} f^{m n} \\
& -\frac{\hbar^{2}(n+m)^{2}}{16 \alpha \operatorname{ch}^{2}(x / 2)} f^{m n}+\frac{\hbar^{2}}{2 \mu}\left\{\begin{array}{c}
m^{2} \\
n^{2}
\end{array}\right\} f^{m n}+\mathcal{V} f^{m n},
\end{aligned}
$$


where $D f^{m n}=(1 /|\operatorname{sh} x|)(\partial / \partial x)\left(|\operatorname{sh} x| \partial f^{m n} / \partial x\right)+\partial^{2} f^{m n} / \partial x^{2}$, and $\mathcal{V}$ is a dilatationsstabilizing potential depending on $q$. As for purely incompressible motion, there exist both bounded and continuous spectra depending on the relationship between quantum numbers $n, m$.

Acknowledgments. It is a great pleasure for authors to thank the Organizers of the XXII Workshop on Geometrical Methods in Physics (June 29 - July 5, 2003, Białowieża, Poland), especially Professor Anatol Odzijewicz, for the possibility of participation in this Conference and their warm hospitality during it. This work has been partially supported by the Committee of Scientific Research's (KBN) grant 8T07A04720.

\section{References}

[1] Hehl F W, Kerlick G D and Van der Heyde P, Physical Review D10 (1974), 1066.

[2] Hehl F W, Lord E A and Ne'eman Y, Hadron Dilatation, Shear and Spin as Components of the Intrinsic Hypermomentum Current and Metric-Affine Theory of Gravitation, Physics Letters 71B (1977), 432.

[3] Ivanenko D D, Pronin P J and Sardanashvily C A, Gauge Theory of Gravitation, Naukova Dumka, Kyiv, 1985 (in Russian).

[4] Sławianowski J J, U(2,2)-Invariant Spinorial Geometrodynamics, Reports on Mathematical Physics 38, 3 (1996) 375-397.

[5] Sławianowski J J, U(2,2)-Symmetry as a Common Basis for Quantum Theory and Geometrodynamics, International Journal of Theoretical Physics 37, 1 (1998), 411-420.

[6] Sławianowski J J, Internal Symmetries of Geometrodynamical Models, Reports on Mathematical Physics 48, 1/2 (2001), 103-114.

[7] Arnold V I, Mathematical Methods of Classical Mechanics, Springer Graduate Texts in Mathematics, 60, Springer-Verlag, New York, 1978.

[8] Bogojavlenski O I, Methods of Qualitative Theory of Dynamical Systems in Astrophysics and Gas Dynamics, Nauka, Moscow, 1980 (in Russian), english translation: Springer, BerlinHeidelberg-New York, 1985.

[9] Eringen A C, Mechanics of Micromorphic Continua, in: Proc. of the IUTAM-Symposium (ed. Kröner E), Freudenstadt and Stuttgart (1967), 18, Springer, Berlin-Heidelberg-New York, 1968, 18-33.

[10] Sławianowska A K and Sławianowski J J, Quantization of Affinely Rigid Body in $N$ Dimensions, Reports on Mathematical Physics 29, 3 (1991), 297-320.

[11] Sławianowski J J, Affinely Rigid Body and Hamiltonian Systems on GL(n,R), Reports on Mathematical Physics 26, 1 (1988), 73-119.

[12] Sławianowski J J and Kovalchuk V, Invariant Geodetic Problems on the Affine Group and Related Hamiltonian Systems, Reports on Mathematical Physics 51, $2 / 3$ (2003), 371-379.

[13] Sławianowski J J, Various Aspects of the Born-Infeld Nonlinearity, Reports on Mathematical Physics 46, 1/2 (2000), 253-260.

[14] Barut A O, Božić M and Marić Z, The Magnetic Top as a Model of Quantum Spin, IC/90/460, Annals of Physics 214, 1 (1992), 53-83. 